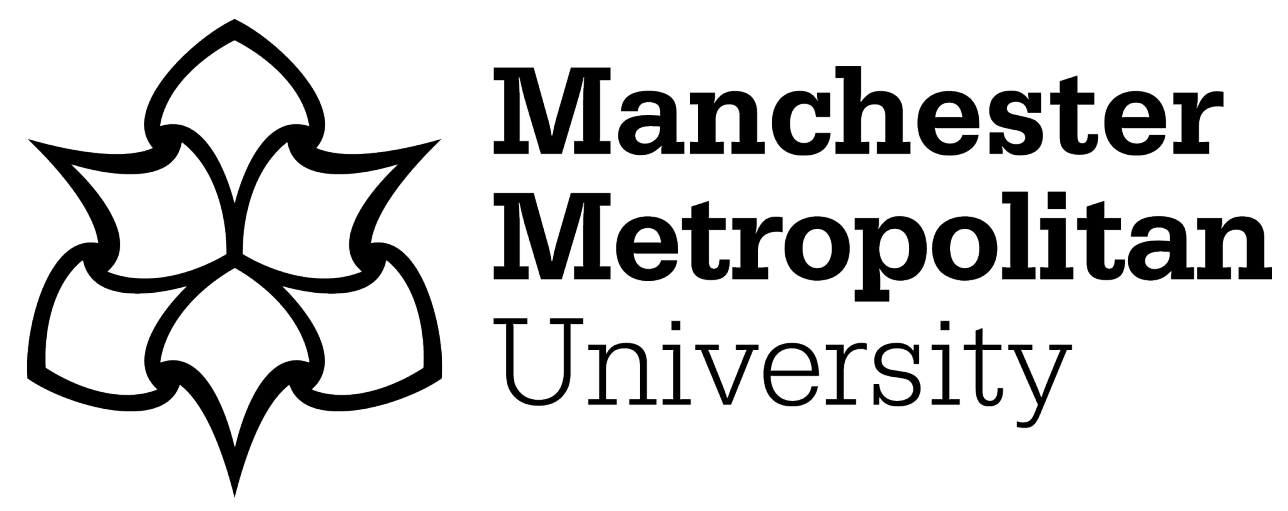

Sheen, KA ORCID logoORCID: https://orcid.org/0000-0002-6326-3241, Luximon, $Y$ and Zhang, J (2017) Reading Task Investigation of the Kindle app in Three Mediums. In: Advances in Physical Ergonomics and Human Factors. AHFE 2017. Advances in Intelligent Systems and Computing, vol 602, 17 July 2017 - 21 July 2017, Los Angeles, USA.

Downloaded from: https://e-space.mmu.ac.uk/625472/

Publisher: Springer

DOI: https://doi.org/10.1007/978-3-319-60825-9_38

Please cite the published version 


\title{
Reading Task Investigation of the Kindle App in Three Mediums
}

\author{
Kimberly Anne Sheen ${ }^{1}$, Yan Luximon ${ }^{1, *}$ and Jiaxin Zhang ${ }^{1}$ \\ ${ }^{1}$ School of Design, The Hong Kong Polytechnic University, Hung Hom, Kowloon, \\ Hong Kong SAR \\ kimberly.sheen@connect.polyu.hk, yan.luximon@polyu.edu.hk, \\ jx.zhang@connect.polyu.hk
}

\begin{abstract}
E-textbooks are often considered the future of textbooks but the current capabilities and implications of app-based textbooks and their corresponding technology are not well outlined. The goal in this study was to understand the effects of the change in medium on the academic reading task, student perception of the devices and components, and identify issues surrounding two in-app components. Students completed four reading tasks in three different size mobile devices and a paper control. The experiment also consisted of a between-subject study where students were asked to use the highlighting or annotation component while reading. Results showed that the devices and components actually changed the way the students interact with their reading. Also, students were generally unhappy with the in-app components and smallest sized device. This information is useful to identify the effects of e-textbook apps on reading behavior, which can be applied to improve the design of future e-textbooks.
\end{abstract}

Keywords: Electronic Textbooks · Textbook Design · Interface Design · Kindle App $\cdot$ Experiment

\section{Introduction}

Education is slowly shifting to new practices, especially when it comes to textbooks. While some universities across the world are hesitant to move to electronic textbooks, others are moving forward with electronic textbook adoption [1]. McFadden predicts that within the next few of years, tablets will take the place of other forms of personal computers to become the primary computing platform in academia [2]. Some of the new mobile devices are attempting to straddle this line with their marketing and technology, such as the iPad Pro, Microsoft Surface, and the Lenovo Yoga.

This shift leads us to the core research question, which drove this study. What effect does screen size of the most frequently used mobile technology have on student academic reading behavior? Past research has given us some insight into this area, but leaves much to be desired in information on some important aspects such as an investigation into how supporting activities, such as highlighting and notetaking, change.

Research based on leisure reading in electronic forms do not necessarily apply to academic reading because academic reading requires higher levels of concentration and 
the ability to infer and deduct the proper aspects of the text so that material may be fully comprehended and recalled later [3,4]. This recall is vital to the task of academic reading because of the academic performance measures such as papers and exams [5]. It is also important to remember that students do not tend to read for school without any supporting activities, such as highlighting and notetaking.

Past research has told us that moving to the electronic form of textbooks does not have a negative impact in comprehension, but some aspects resulting from the switch to the digital medium such as sentence splitting may in fact negatively influence comprehension [6]. Since working memory is limited, the complexity of the learning task will increase the cognitive load on the working memory and thus impeded the student's learning of the material [7]. It is vital to acknowledge cognitive load as there is a correlation between working memory and academic achievement [8]. Research has also shown that the visual demands of electronic texts, especially hypertext, increases cognitive load [9]. Past research has found the different types of e-readers did not have an effect on students' learning of the material [10]. In fact, teachers have reported benefits in some of the different functionalities available through electronic textbooks, such as the ability for students to take notes they can refer back to and the built in dictionary components [11].

While research showed that there is little difference in student comprehension of the material, student behavior has been shown to change. Woody, et al. found that students are more likely to use certain aspects of the physical textbook, such as reading summaries or answering questions, than those included in electronic textbooks [12]. And several studies have found that students spend more time with an electronic textbook than the printed counterpart $[13,14]$. Yet, this same finding regarding time spent reading is discounted in other literature [15].

Many of the studies listed above compare only a physical textbook to an electronic addition or various e-readers to each other. For example, apps such as the Kindle app has been studied before, but in relation to other reading applications [16]. So, this study evaluates one electronic textbook on the Kindle app using three mobile devices commonly used by students at the university and paper control which was the same size and format as the largest mobile device. In addition, two of the commonly used supporting activity components, the highlighting and annotation components, were also investigated to identify any changes in study behavior.

\section{$2 \quad$ Method}

\subsection{Participants}

A total of 92 students participated in this research. 51 of those students were female while 41 were male. The average age of participants was 25 years old.

There were a total of three qualifying factors for participation in the experiment. First, the participant had to be a current student at the university. Students were chosen as experiment participants because of their familiarity with academic texts. Second, students were required to have normal or corrected vision. Finally, students were required to have a native language other than English and pass a pretest. Non-native English speakers were chosen as the target group for the study as current research on 
electronic textbooks does not always take into account non-native language users of the books. With more and more students studying abroad and universities offering courses in languages other than the native language their students use, this is an important user group. Education level was not considered a qualifying factor for this experiment as a student's reading level did not necessarily coincide with their education level. Instead, groups were balanced by pretest results. If a student performed too poorly on the pretest, they were disqualified from participating in the experiment.

\subsection{Equipment}

An iPhone 6s, iPad mini, and iPad were used during this experiment (see Table 1). The three forms of mobile devices used were chosen based on the prevalence of usage within the university. All devices used the same operating system so as to have the least amount of differences within the app and subsequent interactions. All three devices had the text size, brightness, and layout preset so the conditions were the same across devices. Devices were also presented to students on a stand and they were not allowed to hold the devices or alter the state of the devices except to change the page, take notes, or highlight depending on group assigned.

The Kindle app was chosen as the application, which the textbook would be presented. This was because of ease of access across the devices and previous research into students at the university showed a general familiarity with the app. The textbook chosen for the students was written in English by professors at a foreign language speaking university to be used in their classrooms. Four individual chapters were chosen from the textbook and educational reading experts deemed appropriate for the experiment, as they were similar in length and reading level.

A Sony HDR-PJ440 Handycam was also used to video record the students interacting with the mobile devices during the reading sessions. The video camera was placed on a tripod located behind the left shoulder of the participants.

Table 1. Screen sizes and resolutions of mobile devices.

\begin{tabular}{llll}
\hline Display Features & Mobile & Mini-Tablet & iPad \\
\hline Screen Resolution & $1334 \times 750$ & $1024 \times 768$ & $1024 \times 768$ \\
Screen Size & 4.7 inches & 7.9 inches & 9.7 inches \\
\hline
\end{tabular}

\subsection{Experimental Design}

The findings presented in this paper were discovered during a mixed factorial design experiment, which used four settings. The four settings were using different devices including mobile phone, mini-tablet, normal sized tablet, and a control group who used paper. The paper control was the same size as the normal iPad so as to identify if changes in task behavior were based on the change in medium without confounding factors such as layout and size. The chapter students were asked to read was randomized. Participants read a chapter on all three of the mobile device sizes and the paper. 
In addition, participants were separated into three different groups. 31 Students were in Group A and completed the readings with the three different screen sizes and paper in the four conditions. 31 students in Group B completed the same process but were requested to use the built in highlighting function or to highlight directly on the paper. And the 30 students in Group C also completed the same process but were requested to use the annotation tool while using the devices and take notes directly on paper when using paper.

Each session ranged from one hour to one and a half hours based on the individual's time spent reading. Participants were paid for their time.

\section{$2.4 \quad$ Procedure}

Participants were briefed on the experimental procedure and signed a consent form. After taking a reading comprehension and recall pretest, students were assigned to one of the three groups. Before the students began reading, they were briefly shown how to use the app and any functions they were required to use. They also had the opportunity to try navigating in the book and opening the annotation tool or using the highlighting function.

The student then began the reading assigned to the condition. After each condition, a rest period of three minutes was completed and students filled out a questionnaire regarding their experience during the reading task. Then they were given a post reading test. Following that, the next condition began. After all conditions were completed, students were asked to compare their experiences in all the conditions and report their general impressions and any issues they found.

\section{$3 \quad$ Results}

\subsection{Time Spent Reading}

Before analysis began, normality of the data was assessed. To ensure the normality of the data, outliers were removed. This left 26 participants in Group A, 31 participants in Group B, and 28 participants in Group C. All results related to time spent reading were based on data from these participants.

This study showed that time spent reading changed not only between the paper control and the various mobile device sizes, but also between groups. Table 2 shows the average time spent reading for each condition in words per minute (wpm).

Table 2. Average time spent reading in Word Per Minute for each group and device.

\begin{tabular}{lllll}
\hline Groups & Paper & iPhone 6s & iPad mini & iPad \\
\hline A & 118 & 109 & 121 & 117 \\
B & 96 & 99 & 106 & 104 \\
C & 95 & 98 & 107 & 103 \\
\hline
\end{tabular}


In Group A, where users were only required to finish the readings in each condition, the iPad mini was shown to afford the fastest time for completion of reading but the changes in time spent reading were not as pronounced between the mini, iPad, and paper control. The difference between the least time spent reading and most across all four conditions was $12 \mathrm{wpm}$. The full size iPad and the paper control were the same size and format and the time spent reading in these two conditions only differed by $1 \mathrm{wpm}$.

Groups B and C included a supporting task, highlighting and note taking respectively, during the reading sessions. Conversely to the findings of Group A, the paper control had the longest time spent reading while the iPad mini continued to afford the shortest time spent reading. Once again, the difference between the iPad and the iPad mini was a decrease of less than $4 \mathrm{wpm}$. While Group A showed a difference of more than $12 \mathrm{wpm}$ between the iPhone and the iPad mini, this large difference in time spent reading was reduced in Groups B and C. Instead, there was a 7wpm decrease in Group $\mathrm{B}$ and 9 wpm decrease in Group $\mathrm{C}$ when using the mobile phone.

\subsection{Changes in Reading Behavior}

There were changes in the behavior of students when using components, which support their reading. Overall, when moving to the Kindle App, students took less notes and used the highlighting tool less frequently.

Students frequently reported, during the experiment, that the platform did not support their habits. Analysis of paper controls found that $73.3 \%$ students in the notetaking group used a more visual notetaking style that is not supported by the app's simple textbook input (see Fig. 1). Also, 16.1\% students in the highlighting group used other marks such as circling or starring to help identify the importance of the material in addition to simple highlighting (see fig. 2). 


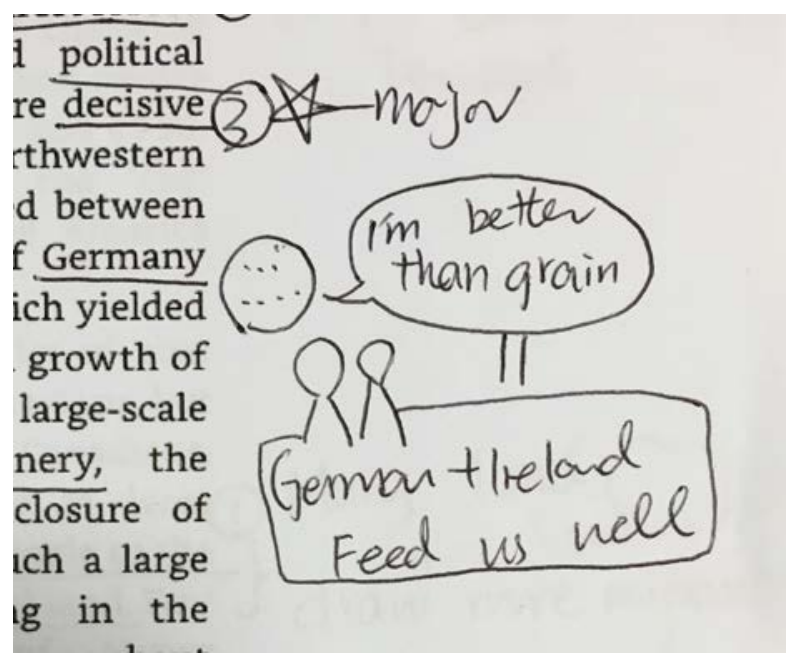

Figure 1. Example of notetaking behavior not supported by Kindle app.
Prior to the presidential election 108,54 percent of respondents in a $\mathrm{N}$. 11 said they tended not to trust $t$ ] neral (30 percent trusted) and 46 F ey did not trust television ( 36 perce iernet news sites were trusted by 41 rcent not trusted). Radio tended to $\dot{c}$ percent saying that they tend to cent distrusted). But a Gallup poll i ind that only 21 percent of respon. at deal or quite a lot of confidence zvision news, a drop to a new lo cent in 2011. It now ranked 11th a1

Figure 2. Example of highlighting behavior that is not supported by Kindle App.

\subsection{Issues Identified}

Several issues were identified during the experiment through facilitator observation and student report after each task. Some of these were related solely to the specific mobile device and others were found across all of the mobile devices. 
Device specific issues were found on the iPhone 6s. This was due to the small screen size. Students frequently reported issues reading the material due to the limited information on the screen. Students also reported difficulties of taking notes and highlighting. The issues of taking notes were related to the small size of the keyboard input. The issues related to highlighting were frequently related to the increased sentence splitting caused by the small screen size. Students would have to highlight text on two different pages and reported this as being difficult and often time consuming.

Issues with the in-app components used in both Groups B and C were reported across devices. Highlighting was reported as difficult for students to complete without using more than one highlighting movement to cover the complete sentence. Similar to what the students reported, the facilitator also observed student issues when they attempted to make an existing highlighted section longer or shorter and at times ended up completely removing the highlighted section and started again. Students also reported that they often went back or forward a page while attempting to highlight a passage.

Students also struggled frequently with the annotation tool. Students reported that the keyboard input was not ideal for inputting their notes. Many students reported frustration with the fact that they could not move the textbox popup so that they could see the text they were referencing. Instead they had to spend more time opening and closing the textbox repeatedly until they could edit their notes to their satisfaction. In addition, the facilitator observed many students getting confused when attempting to access the annotation component. When they would select a word or phrase, students would initially look at the larger dictionary, thesaurus, Wikipedia boxes that pop up bellow the toolbar. Some students even attempted to select those options out of reflex. Students also showed frustration with the way the notetaking icon was represented. Several students deleted the note to try and select a phrase once again to only have the same icon appear. A few of these students then used the highlighting component to identify the corresponding phrase.

\section{Discussion}

In general, the time spent reading while using physical text in Group A did not change much from the electronic version of the text. Findings from Group A showed that time reading was shorter in paper than most of the electronic mediums, which is supported by previous research [14]. Still, reading was completed faster on the iPad mini, which is supported by contradictory research that found that time spent reading decreased when using the electronic version of texts [15]. This trend of shorter time spent reading in the paper medium was not sustained in Groups B and C where the components were introduced. This discrepancy is possibly related to the increase in highlights and notes that students in those groups took in the paper form. Since students reported struggling with these functions or that the components did not support their habits, the time they saved by using them less frequently is likely the cause of this change in time spent reading.

While past surveys have shown that hundreds of thousands of students wish to be able to take notes or highlight in their electronic textbooks [17], these components are not yet optimized for students in the Kindle app. In fact, these features, which are considered essential, have yet to be perfected in any e-reader [18]. And while all the 
students in Group B were able to use the highlighting function in this study, although with difficulty, past research found that less than $80 \%$ of Kindle app users on an iPad 3 were able to use the highlight function within 1 minute [16].

\section{Conclusion}

The findings from this study showed that student academic reading behavior does change not only when moving from the print to electronic medium, but there are also changes when moving between different sized mobile devices and when using different components of those electronic textbooks. The time spent reading was less for students reading in a print medium in the same size and format as the electronic textbook. Yet, this increase in speed is lost when students begin using supporting activities such as highlighting or notetaking. Students tended to do more highlighting and notetaking in terms of number of words and different styles in paper format. In addition, students struggled to use the functions in general and found them frustrating. There was a marked increase in time spent reading when moving to the mobile phone condition in all groups and students reported the least satisfaction with reading on the device in general due to their greater difficulties in reading the material and using the functions.

Acknowledgments. The authors would like to thank The Hong Kong Polytechnic University for their support. This research was supported by the Research Grants Council for the Hong Kong PhD Fellowship Scheme (1-904Z).

\section{References}

1. Chesser, W. D.: The e-textbook revolution. Library technology reports 47(8), 28 (2011)

2. McFadden, C.: Are textbooks dead? Making sense of the digital transition. Publish. Res. Q. 28(2), 93--99 (2012)

3. Merritt, J.E.: Developing Fluent Reading. Open University Press (1977)

4. Phillips, B.J., Phillips, F.: Sink or skim: Textbook reading behaviors of introductory accounting students. Issues in Accounting Education 22(1), 21--44 (2007).

5. Simpson, M.L., Nist, S.L.: An update on strategic learning: It's more than textbook reading strategies. J Adolesc. Adult Lit. 43(6), 528--541 (2000)

6. Dillon, A.: Reading from paper versus screens: A critical review of the empirical literature. Ergonomics 35(10), 1297--1326 (1992)

7. Sweller, J.: Cognitive load theory, learning difficulty, and instructional design. Learning Instr. 4(4), 295--312 (1994)

8. Yuan, K., Steedle, J., Shavelson, R., Alonzo, A., Oppezzo, M.: Working memory, fluid intelligence, and science learning. Educ. Res. Rev-Neth. 1(2), 83--98 (2006)

9. DeStefano, D., LeFevre, J.A.: Cognitive load in hypertext reading: A review. Comput. Hum. Behav. 23(3), 1616--1641 (2007)

10.Weisberg, M.: Student attitudes and behaviors towards digital textbooks. Publish. Res. Q., 27(2), 188--196 (2011)

11.Demski, J.: Ell to go: Two schools transform their Ell programs by giving students aroundthe-clock access to some of the latest mobile devices. THE Journal (Technological Horizons In Education) 38(5), 28 (2011)

12. Woody, W.D., Daniel, D.B., Baker, C.A.: E-books or textbooks: Students prefer textbooks. Comput. Educ. 55(3), 945--948 (2010) 
13.Connell, C., Bayliss, L., Farmer, W.: Effects of eBook readers and tablet computers on reading comprehension. International Journal of Instructional Media 39(2), 131--141 (2012)

14.Daniel, D.B., Woody, W.D.: E-textbooks at what cost? Performance and use of electronic v. print texts. Comput. Educ. 62, 18--23 (2013)

15. Shepperd, J.A., Grace, J.L., Koch, E.J.: Evaluating the electronic textbook: is it time to dispense with the paper text?. Teach. Psychol. 35(1), 2--5 (2008)

16.Jardina, J.R., Chaparro, B.S.: Usability, engagement, and satisfaction of two e-textbook applications. In: Proceedings of the Human Factors and Ergonomics Society Annual Meeting, pp. 482--486. Sage Publications: Los Angeles (2013)

17. Warren, J.W.: The Progression of Digital Publishing: Innovation and the E-volution of Ebooks. International Journal of the Book 7(4), (2010)

18.Ferguson, J., Giordano, J., Jerome, E., Lipkin, A., Pinnolis, J., Widmer, L.: LTS E-textbook analysis, http://bir.brandeis.edu/bitstream/handle/10192/23919/EtextbookReport_LTS-RISSpring2010.pdf 